\title{
Análise comparativa de métodos preditivos para determinação de propriedades termofísicas de acilgliceróis parciais de cadeia curta
}

\author{
D. S. DAMACENO ${ }^{1}$, R. CERIANI ${ }^{1}$ \\ ${ }^{1}$ Universidade Estadual de Campinas, Departamento de Desenvolvimentos de Processos e Produtos \\ E-mail para contato: rceriani@feq.unicamp.br
}

\begin{abstract}
RESUMO - Nos últimos anos a indústria de óleos e gorduras vem crescendo em larga escala, principalmente por causa do aumento de produção de biocombustíveis. Como consequência, a demanda por propriedades termofísicas tem aumentado e, logo, dados experimentais e a modelagem termodinâmica envolvendo compostos da tecnologia de lipídios estão se tornando cada vez mais temas de pesquisa. Nesse contexto, este trabalho analisou a capacidade preditiva dos modelos de Ceriani et al. (2013), Zong et al. (2010), Marrero e Gani (2001), Tu e Liu (1996), Basařová e Svoboda (1995), Joback e Reid (1987), na predição da pressão de vapor (temperatura de ebulição), temperatura normal de ebulição e entalpia de vaporização de acilgliceróis parciais de cadeia curta (monocaprilina, monocaprina, dicaprilina e dicaprina). A comparação foi feita com base nos dados experimentais publicados recentemente por Damaceno et al. (2014). Baseado na análise da capacidade preditiva dos métodos selecionados, esse estudo recomenda os modelos de Ceriani et al. (2013), Zong et al. (2010) e Marrero e Gani (2001) para a estimativa das propriedades termofísicas selecionadas.
\end{abstract}

\section{INTRODUÇÃO}

Óleos e gorduras são uma fonte nutricional relevante de energia, de ácidos graxos essenciais, de vitaminas e de antioxidantes naturais, além da demanda recente como matéria-prima para produção de biodiesel. Em sua grande maioria, passam por algum tipo de processamento antes de serem destinados ao consumo humano. Além disso, derivados de óleos vegetais como ácidos graxos, ésteres graxos, triacilgliceróis, diacilgliceróis e monoacilgliceróis são importantes produtos oleoquímicos, de aplicação nas indústrias de alimentos e química. Neste contexto, o conhecimento e a descrição do comportamento de propriedades termofísicas de compostos graxos em função das diferentes variáveis de processamento podem ser de grande utilidade prática. No entanto, dados experimentais dessas propriedades na literatura aberta são bastante escassos, principalmente com relação aos acilgliceróis parciais. Por exemplo, no caso da temperatura de ebulição, até recentemente encontravam-se na literatura aberta apenas 6 dados experimentais a $0,13 \mathrm{kPa}$ para 6 diferentes monoacilgliceróis (MAG) e nenhum dado para diacilgliceróis (DAG). Já para os triacilgliceróis (TAG), há em torno de 226 dados de reportados (Ceriani et al., 2013; Damaceno et al., 2014). Devido a essa carência, Damaceno et al. (2014) desenvolveram um trabalho experimental para determinação de dados de temperatura de ebulição à baixas pressões $(1,0 \mathrm{kPa}$ a $13,2 \mathrm{kPa})$ para quatro acilgliceróis parciais de cadeia curta, 


\section{9 a 22 de outubro de 2014 \\ Florianópolis/SC}

denominados monocaprilina, monocaprina, dicaprilina e dicaprina, utilizando a técnica da calorimetria diferencial exploratória (DSC).

A literatura apresenta diversos trabalhos de aplicação do conceito de contribuição de grupos para a determinação de propriedades termofísicas de compostos puros, tais como: pressão de vapor (Ceriani et al., 2013), entalpia de vaporização (Ceriani et al., 2013; Basařová e Svoboda, 1995; Marrero e Gani, 2001; Tu e Liu, 1996), temperatura normal de ebulição (Marrero e Gani, 2001; Joback e Reid, 1987), viscosidade (Ceriani et al., 2011; Díaz-Tovar et al., 2011), propriedades críticas (Marrero e Gani, 2001; Joback e Reid, 1987), tensão superficial e densidade (Díaz-Tovar et al., 2011), dentre outros. Alguns desses trabalhos foram desenvolvidos especificamente para compostos graxos, como os modelos de Ceriani et al. (2013), Ceriani et al. (2011) e Díaz-Tovar et al. (2011). Além disso, há outros métodos, como o de Zong et al. (2010) que prediz dados de pressão de vapor (temperatura de ebulição), entalpia de vaporização, dentre outras propriedades para acilgliceróis baseado num conceito que os autores denominaram fragment-based approach.

Do que foi exposto acima, este trabalho teve como objetivo analisar a capacidade preditiva dos modelos de Ceriani et al. (2013), Zong et al. (2010), Marrero e Gani (2001), Tu e Liu (1996), Basařová e Svoboda (1995), Joback e Reid (1987) na determinação de dados de pressão de vapor (temperatura de ebulição), temperatura normal de ebulição e entalpia de vaporização de acilgliceróis parciais de cadeia curta. Para efeito de comparação foram considerados os dados experimentais das temperaturas de ebulição da monocaprilina, monocaprina, dicaprilina e dicaprina, em uma faixa de pressão de 1,0 kPa a 13,2 kPa, e as correlações geradas pelos autores (descritas no item 2.1).

\section{METODOLOGIA}

\subsection{Modelagem da pressão de vapor e entalpia de vaporização segundo Damaceno et al. (2014)}

O modelo ajustado por Damaceno et al. (2014) para a pressão de vapor em função da temperatura foi baseado na equação DIPPR (Design Institute for Physical Properties):

$$
\ln p=A+\frac{B}{T}+C \ln T+D T
$$

onde $p(\mathrm{kPa})$ é a pressão de vapor, $T(\mathrm{~K})$ é a temperatura de ebulição, e $A, B, C$ e $D$ são os parâmetros obtidos pela regressão dos dados experimentais (Damaceno et al., 2014) em conjunto com a temperatura normal de ebulição $\left(T_{b}\right)$ e ponto crítico $\left(T_{c}, P_{c}\right)$, ambos calculados pelo método de contribuição de grupos de Marrero e Gani (2001), como sugerido por Ceriani et al. (2013).

Para a entalpia de vaporização $\left(\Delta H_{v a p}\right)$, Damaceno et al. (2014) consideraram a equação de Clausius-Clapeyron (Poling et al., 2004) com uma correção para extensão da dependência da entalpia de vaporização com a derivada da pressão de vapor em relação à temperatura até o ponto crítico (Equação 2). Os valores calculados para $T_{c}(\mathrm{~K})$ e $P_{c}(\mathrm{kPa})$ por Marrero e Gani (2001) para monocaprilina, monocaprina, dicaprilina e dicaprina são: 771,15 K e 2252,3 kPa, 792,13 K e 1940,3 $\mathrm{kPa}, 836,43 \mathrm{~K}$ e $1350,5 \mathrm{kPa}$ e $867,37 \mathrm{~K}$ e $1168,4 \mathrm{kPa}$, respectivamente. 


$$
\Delta H_{v a p}=R\left(-B+C T+D T^{2}\right)\left(1-T / T_{c}\right)^{p / P_{c}}
$$

onde $P_{c}(\mathrm{kPa})$ e $T_{c}(\mathrm{~K})$ são a pressão e temperatura críticas, respectivamente.

A Tabela 1 traz os parâmetros $A, B, C$ e $D$ das Equações 1 e 2 reportados por Damaceno et al. (2014). Os desvios médios relativos obtidos pelos autores no ajuste desses parâmetros entre temperatura de ebulição calculada e a experimental variaram entre 0,5 e $0,9 \%$

Tabela 1 - Parâmetros $A, B, C$ e $D$ para predição de $p(\mathrm{kPa})$ e $\Delta H_{v a p}(\mathrm{~J} / \mathrm{mol})$ segundo Damaceno et al. (2014)

\begin{tabular}{lcccc}
\hline \multirow{2}{*}{ Composto } & \multicolumn{4}{c}{ Constantes } \\
\cline { 2 - 5 } & $A$ & $B$ & $C$ & $D$ \\
\hline Monocaprilina & 43,596 & $-13753,5$ & $-1,327$ & $-0,0119$ \\
Monocaprina & 45,274 & $-14516,9$ & $-1,347$ & $-0,0132$ \\
Dicaprilina & 48,264 & $-16351,6$ & $-1,382$ & $-0,0146$ \\
Dicaprina & 48,451 & $-17363,6$ & $-1,295$ & $-0,0146$ \\
\hline
\end{tabular}

\subsection{Modelos preditivos de propriedades termofísicas}

A seguir são apresentados os modelos preditivos para temperatura normal de ebulição $\left(T_{n b}\right)$, pressão de vapor $(p)$ e entalpia de vaporização $\left(\Delta H_{\text {vap }}\right)$ avaliados nesse trabalho. As fórmulas químicas dos compostos considerados são: monocaprilina ou 1-octanoil glicerol $\left(\mathrm{C}_{11} \mathrm{H}_{22} \mathrm{O}_{4}\right)$, monocaprina ou 1-decanoil glicerol $\left(\mathrm{C}_{13} \mathrm{H}_{26} \mathrm{O}_{4}\right)$, dicaprilina ou 1,2-dioctanoil glicerol $\left(\mathrm{C}_{19} \mathrm{H}_{36} \mathrm{O}_{5}\right)$ e dicaprina ou 1,2-didecanoil glicerol $\left(\mathrm{C}_{23} \mathrm{H}_{44} \mathrm{O}_{5}\right)$. A divisão dos grupos funcionais de cada modelo considerado nesse trabalho para os acilgliceróis parciais está discriminada na Tabela 2.

Tabela 2 - Divisão em grupos funcionais dos acilgliceróis parciais segundo cada modelo considerado.

\begin{tabular}{lllllllllll}
\hline \multicolumn{1}{c}{ Modelo } & \multicolumn{1}{c}{ CH3 } & $\mathrm{CH} 2$ & $\mathrm{CH}$ & $\mathrm{OH}$ & $\mathrm{CH} 2 \mathrm{COO}$ & $\mathrm{CHCOO}$ & $\mathrm{COO}$ & $\mathrm{CH} 2 \mathrm{CHCH} 2$ \\
\hline Ceriani et al. (2013) & $p$ & $\mathrm{X}$ & $\mathrm{X}$ & & $\mathrm{X}$ & & & $\mathrm{X}$ & $\mathrm{X}$ \\
Zong et al. (2010) & $p e \Delta H_{\text {vap }}$ & & & & & & & & \\
Marrero e Gani (2001) & $\Delta H_{\text {vap }} e T_{n b}$ & $\mathrm{X}$ & $\mathrm{X}$ & $\mathrm{X}$ & $\mathrm{X}$ & $\mathrm{X}$ & $\mathrm{X}$ & & \\
Tu e Liu (1996) & $\Delta H_{\text {vap }}$ & & & & & & & & \\
Basařová e Svoboda (1995) & $\Delta H_{\text {vap }}$ & $\mathrm{X}$ & $\mathrm{X}$ & $\mathrm{X}$ & $\mathrm{X}$ & & & $\mathrm{X}$ & \\
Joback e Reid (1987) & $T_{n b}$ & $\mathrm{X}$ & $\mathrm{X}$ & $\mathrm{X}$ & $\mathrm{X}$ & & & $\mathrm{X}$ & \\
\hline
\end{tabular}

Temperatura normal de ebulição $\left(T_{n b}\right.$, em K): $\mathrm{O}$ modelo avaliado para $T_{n b}$ foi o proposto por Joback e Reid (1987) (Equação 3).

$$
T_{n b}=198+\sum_{k} N_{k}\left(t_{n b} k\right)
$$

onde: $N_{k}$ é o número de contribuições e $t_{n b} k$ é a contribuição de cada grupo.

Vale lembrar que o modelo de Marrero e Gani (2001) (Equação 4) foi utilizado por Damaceno 
et al. (2014) na predição da $\underline{T}_{n \underline{b}}$ que foi acrescida ao banco de dados experimental juntamente com o ponto crítico para então se obter os parâmetros reportados na Tabela 1.

$$
\exp \left(T_{n b} / T_{n b 0}\right)=\sum_{i} N_{i} T_{n b 1 i}+\sum_{j} M_{j} T_{n b 2 j}+\sum_{k} O_{k} T_{n b 3 k}
$$

onde: $N_{i}, M_{j}$ e $O_{k}$ é o número de contribuições e $T_{n b 1 i}, T_{n b 2 j}$ e $T_{n b 3 k}$ são as contribuições de cada grupo, e $T_{n b 0}$ é uma constante.

Pressão de vapor $(p)$ : foram avaliados o modelo de Ceriani et al. (2013) (Equação 5), que se baseia no método de contribuição de grupos (Tabela 2) e de Zong et al. (2010) (Equação 6) que utiliza o método de fragmentação das classes de compostos químicos, nesse caso: MAG/DAG/ C8:0/C10:0. Para a avaliação da capacidade preditiva das duas equações, foram considerados os desvios gerados na estimativa da temperatura de ebulição a uma dada pressão, visto que essa é a variável utilizada na simulação computacional de colunas de separação líquido-vapor (Ceriani, 2005).

$$
\ln (p)=A+\frac{B}{T}+C \cdot \ln (T)
$$

onde $p$ é a pressão de vapor em $\mathrm{Pa}, T$ é a temperatura em $\mathrm{K}$, e $A, B$ e $C$ são parâmetros obtido pelo somatório de cada grupo.

$$
\log (p)=\frac{-\Delta G_{\theta}^{v a p}}{R \theta \ln 10}+\frac{\Delta H_{\theta}^{v a p}}{R \ln 10}\left(\frac{1}{\theta}-\frac{1}{T}\right)
$$

onde $R$ é a constante dos gases, $\theta$ é a temperatura de referencia em $298,15 \mathrm{~K}, \Delta H_{\theta}{ }^{\text {vap }}$ é a entalpia de vaporização e $\Delta G_{\theta}{ }^{\text {vap }}$ é a energia livre de Gibbs.

Entalpia de vaporização $\left(\Delta H_{v a p}\right)$ : pode ser estimada por cinco diferentes métodos, sendo que dois deles determinam $\Delta H_{\text {vap }}$ apenas a 298,15 K. São eles, os modelos de Marrero e Gani (2001) (Equação 7) e Zong et al. (2010) (Equação 8).

$$
\left(\Delta H_{v a p}-\Delta H_{v a p 0}\right)=\sum_{i} N_{i} H_{v a p 1 i}+\sum_{j} M_{j} H_{v a p 2 j}
$$

onde $\Delta H_{v a p}$ é a entalpia de vaporização a $298,15 \mathrm{~K}, N_{i}$ e $M_{j}$ é o número de contribuições e $H_{n b l i}$ e $H_{n b 2 j}$ são as contribuição de cada grupo e $H_{v a p 0}$ é uma constante.

$$
\Delta H_{\theta}^{v a p}=\sum_{A} N_{f r a g, A} \Delta H_{\theta, A}^{v a p}
$$
298,15 .

onde $N_{f r a g, A}$ é o número de fragmentos, $\Delta H_{\theta, A}{ }^{v a p}$ é a entalpia de vaporização de cada fragmento a

Já os modelos de Ceriani et al. (2013) (Equação 9), Tu e Liu (1996) (Equação 10) e Basařová e Svoboda (1995) (Equação 11) determinam as entalpias de vaporização em qualquer temperatura da curva de vaporização.

$$
\Delta H_{v a p}=R(-B+C T)\left(1-T / T_{c}\right)^{p / P_{c}}
$$


onde: $P_{c}(\mathrm{kPa})$ e $T_{c}(\mathrm{~K})$ são a pressão e temperatura críticas, $B$ e $C$ são obtidos pelos somatórios das contribuição dos grupos.

$$
\left.\Delta H_{v a p}=\sum_{i} N_{i} \mid a_{i}\left(1-T / T_{c}\right)^{1 / 3}+b_{i}\left(1-T / T_{c}\right)^{2 / 3}+c_{i}\left(1-T / T_{c}\right)\right\rfloor
$$

onde $N_{i}$ é o número de fragmentos, $a_{i}, b_{i}$ e $c_{i}$ são os valores de cada contribuição

$$
\Delta H_{v a p}=A\left(1-T / T_{c}\right)^{\alpha} \exp \left[-\alpha\left(1-T / T_{c}\right)\right]
$$

onde $A$ e $\alpha$ são os obtidos pelos somatórios de cada contribuição.

\section{RESULTADOS}

A partir das Equações 1 e 2 e dos parâmetros apresentados na Tabela 1 foi possível determinar a $T_{n b}$ e a $\Delta H_{v a p}$ a $298,15 \mathrm{~K}$ para os MAG e DAG considerados nesse trabalho (Tabela 3). A Tabela 3 também apresenta os valores calculados pelos modelos de Joback e Reid (1987) e Marrero e Gani (2001). A $T_{n b}$ apresentou $169,53 \mathrm{~K}$ e $26,39 \%$ de desvios médios absoluto (DMA) e relativo (DMR) para o modelo de Joback e Reid (1987), respectivamente. As discrepâncias de 102 K a 298 K entre os valores preditos pelo modelo de Joback e Reid (1987) e de Marrero e Gani (Damaceno et al., 2014) é notória. Cunico et al. (2013) também observaram que o modelo de Joback e Reid (1987) prediz valores divergentes em comparação com valores experimentais de $T_{n b}$ para outros compostos graxos.

Tabela 3 - Temperatura normal de ebulição $\left(T_{n b}\right)$ para os mono- e diacilgliceróis considerados nesse trabalho

\begin{tabular}{lcc}
\hline \multicolumn{1}{c}{ Composto } & Joback e Reid (1987) & Marrero e Gani segundo (Damaceno et al. (2014) \\
\hline Monocaprilina & 693,22 & 591,39 \\
Monocaprina & 738,98 & 612,63 \\
Dicaprilina & 842,3 & 656,58 \\
Dicaprina & 933,82 & 687,58 \\
\hline
\end{tabular}

As entalpias de vaporização a 298,15 K obtidas pelos modelos de Marrero e Gani (2001) e Zong et al. (2010) estão reportadas na Tabela 4, em conjunto com os valores calculados pela Equação 2 com os parâmetros da Tabela 1. De uma forma geral, pode-se observar que o modelo de Zong et al. (2010) é capaz de predizer os valores de Damaceno et al. (2014) com maior acurácia.

Tabela 4 - Entalpia de vaporização a 298,15 K para os MAG e DAG considerados nesse trabalho

\begin{tabular}{lccccc}
\hline \multirow{2}{*}{ Composto } & Marrero e Gani & $\begin{array}{c}\left(\Delta H_{\text {vap }} \mathrm{kJ} / \mathrm{mol}\right) \\
\text { Zong } \text { et al. } \\
(2001)\end{array}$ & $\begin{array}{c}\text { Damaceno } \text { et al. } \\
(2010)\end{array}$ & $\begin{array}{c}\text { Mesvio relativo }(\%) \\
\text { Marrero e Gani } \\
(2001)\end{array}$ & $\begin{array}{c}\text { Zong } \text { et al. } \\
(2010)\end{array}$ \\
\hline Monocaprilina & 123,8 & 91,9 & 102,2 & 21,2 & 10,0 \\
Monocaprina & 133,6 & 94,7 & 107,6 & 24,2 & 12,0 \\
Dicaprilina & 154,2 & 103,8 & 121,7 & 26,7 & 14,7 \\
Dicaprina & 173,9 & 109,3 & 130,4 & 33,3 & 16,2 \\
\hline & & $\mathbf{D M R}$ & $\mathbf{2 8 , 1}$ & $\mathbf{1 3 , 2}$ \\
\cline { 3 - 5 }
\end{tabular}

Os valores experimentais de temperatura de ebulição reportados por Damaceno et al. (2014) foram preditos pelos modelos de Ceriani et al. (2013) e de Zong et al. (2010). A Tabela 5 apresenta os valores experimentais e os valores preditivos pelos dois modelos, bem como os desvios absolutos (K) 


\section{LCoBe}

19 a 22 de outubro de 2014

Florianópolis/SC

e relativos $(\%)$.

Tabela 5 - Valores experimentais (Damaceno et al., 2014) e preditos (Ceriani et al., 2013; Zong et al., 2010)

\begin{tabular}{|c|c|c|c|c|c|c|c|c|}
\hline \multirow{2}{*}{ Composto } & \multicolumn{2}{|c|}{ Experimental } & \multicolumn{3}{|c|}{ Ceriani et al. (2013) } & \multicolumn{3}{|c|}{ Zong et al. (2010) } \\
\hline & Pressão $(\mathrm{kPa})$ & Temperatura (K) & $T_{\text {calc }}(\mathrm{K})$ & DA $(\mathrm{K})$ & DR $(\%)$ & $T_{\text {calc }}(\mathrm{K})$ & DA $(\mathrm{K})$ & DR $(\%)$ \\
\hline \multirow{9}{*}{ Monocaprilina } & 1,1 & 462,66 & 471,05 & 8,39 & 1,81 & 469,78 & 7,12 & 1,54 \\
\hline & 1,5 & 468,91 & 476,6 & 7,69 & 1,64 & 476,05 & 7,14 & 1,52 \\
\hline & 2,5 & 479,02 & 486,15 & 7,13 & 1,49 & 486,75 & 7,73 & 1,61 \\
\hline & 3,1 & 484,24 & 490,35 & 6,11 & 1,26 & 491,41 & 7,17 & 1,48 \\
\hline & 3,6 & 485,63 & 493,33 & 7,7 & 1,58 & 494,69 & 9,06 & 1,87 \\
\hline & 4,3 & 490,19 & 496,93 & 6,74 & 1,38 & 498,66 & 8,47 & 1,73 \\
\hline & 6,8 & 498,91 & 506,61 & 7,7 & 1,54 & 509,18 & 10,27 & 2,06 \\
\hline & 10,1 & 507,96 & 515,43 & 7,47 & 1,47 & 518,63 & 10,67 & 2,10 \\
\hline & 13,2 & 514,64 & 521,66 & 7,02 & 1,36 & 525,22 & 10,58 & 2,06 \\
\hline \multirow{9}{*}{ Monocaprina } & 1,1 & 478,69 & 483,26 & 4,57 & 0,95 & 485,42 & 6,73 & 1,41 \\
\hline & 1,5 & 482,79 & 489,09 & 6,3 & 1,3 & 491,93 & 9,14 & 1,89 \\
\hline & 2,5 & 491,77 & 499,15 & 7,38 & 1,5 & 503,02 & 11,25 & 2,29 \\
\hline & 3,1 & 497,13 & 503,57 & 6,44 & 1,3 & 507,85 & 10,72 & 2,16 \\
\hline & 3,6 & 498,31 & 506,71 & 8,4 & 1,69 & 511,26 & 12,95 & 2,60 \\
\hline & 4,3 & 501,31 & 510,52 & 9,21 & 1,84 & 515,37 & 14,06 & 2,80 \\
\hline & 6,8 & 511,21 & 520,74 & 9,53 & 1,87 & 526,28 & 15,07 & 2,95 \\
\hline & 10,1 & 519,1 & 530,08 & 10,98 & 2,12 & 536,08 & 16,98 & 3,27 \\
\hline & 13,2 & 524,41 & 536,69 & 12,28 & 2,34 & 542,92 & 18,51 & 3,53 \\
\hline \multirow{9}{*}{ Dicaprilina } & 1,1 & 511,93 & 504,65 & 7,28 & 1,42 & 506,31 & 5,62 & 1,10 \\
\hline & 1,5 & 517,23 & 511,36 & 5,87 & 1,13 & 512,76 & 4,47 & 0,86 \\
\hline & 2,5 & 529,42 & 522,96 & 6,46 & 1,22 & 523,75 & 5,67 & 1,07 \\
\hline & 3,1 & 533,36 & 528,07 & 5,29 & 0,99 & 528,52 & 4,84 & 0,91 \\
\hline & 3,6 & 538,14 & 531,69 & 6,45 & 1,2 & 531,89 & 6,25 & 1,16 \\
\hline & 4,3 & 541,18 & 536,1 & 5,08 & 0,94 & 535,94 & 5,24 & 0,97 \\
\hline & 6,8 & 552,59 & 547,94 & 4,65 & 0,84 & 546,70 & 5,89 & 1,07 \\
\hline & 10,1 & 560,35 & 558,77 & 1,58 & 0,28 & 556,34 & 4,01 & 0,72 \\
\hline & 13,2 & 567,73 & 566,44 & 1,29 & 0,23 & 563,06 & 4,67 & 0,82 \\
\hline \multirow{7}{*}{ Dicaprina } & 1 & 535,66 & 530,43 & 5,23 & 0,98 & 534,43 & 1,23 & 0,23 \\
\hline & 1,1 & 538,32 & 532,61 & 5,71 & 1,06 & 536,50 & 1,82 & 0,34 \\
\hline & 1,5 & 544,06 & 539,86 & 4,2 & 0,77 & 543,38 & 0,68 & 0,13 \\
\hline & 2,5 & 554,25 & 552,41 & 1,84 & 0,33 & 555,08 & 0,83 & 0,15 \\
\hline & 3,1 & 558,74 & 557,94 & 0,8 & 0,14 & 560,17 & 1,43 & 0,26 \\
\hline & 3,6 & 562,37 & 561,88 & 0,49 & 0,09 & 563,76 & 1,39 & 0,25 \\
\hline & 4,3 & 565,39 & 566,66 & 1,27 & 0,22 & 568,09 & 2,70 & 0,48 \\
\hline
\end{tabular}

De forma geral, observa-se que o modelo de Ceriani et al. (2013) foi aquele que apresentou os menores desvios relativos, com exceção da dicaprina para o qual o modelo de Zong et al. (2010) obteve menores desvios. Com relação à entalpia de vaporização, Damaceno et al. (2014) reportam os valores experimentais apresentados na Tabela 6. A tabela também apresenta as faixas de valores calculados pelos modelos de Ceriani et al. (2013), Tu e Liu (1996) e Basařová e Svoboda (1995) para 
as faixas de temperaturas de ebulição reportados na Tabela 5. Observou-se que o modelo de Tu e Liu (1996) superestima os valores de entalpia de vaporização, já os modelos de Ceriani et al. (2013) e Basařová e Svoboda (1995) os subestimam, porém fica claro que o modelo de Basařová e Svoboda (1995) foi o que apresentou os desvios absolutos maiores (31 kJ/mol, em média).

Tabela $6-\Delta H_{v a p}$ experimental (Damaceno et al., 2014) e o mínimo e o máximo de $\Delta H_{v a p}$ dos modelos preditos

\begin{tabular}{lccccc}
\hline \multirow{2}{*}{ Composto } & Faixa de temperatura & \multicolumn{4}{c}{$\Delta H_{\text {vap }}(\mathrm{kJ} / \mathrm{mol})$} \\
\cline { 3 - 6 } & $(\mathrm{K})$ & $\begin{array}{c}\text { Damaceno } \text { et al. } \\
(2014)\end{array}$ & $\begin{array}{c}\text { Ceriani } \text { et al. } \\
(2013)\end{array}$ & $\begin{array}{c}\text { Tu e Liu } \\
(1996)\end{array}$ & $\begin{array}{c}\text { Basařová e } \\
\text { Svoboda (1995) }\end{array}$ \\
\hline Monocaprilina & $462,66-514,64$ & 96,0 & $106,5-96,2$ & $108,8-99,7$ & $77,0-66,3$ \\
Monocaprina & $478,69-524,41$ & 111,7 & $105,9-97,0$ & $115,6-107,3$ & $79,2-69,5$ \\
Dicaprilina & $511,93-567,73$ & 107,0 & $98,5-90,0$ & $124,1-113,8$ & $86,4-75,2$ \\
Dicaprina & $535,66-565,39$ & 124,1 & $102,2-97,9$ & $137,8-131,9$ & $91,1-84,7$ \\
\hline
\end{tabular}

A Figura 1 mostra o comportamento da pressão de vapor de acordo com os modelos de Ceriani et al. (2013) e Zong et al. (2010), e da entalpia de vaporização, de acordo com os modelos de Ceriani et al. (2013), Tu e Liu (1996) e Basařová e Svoboda (1995) em função da temperatura reduzida $\left(T_{r}=T / T_{c}\right)$ para a monocaprilina, calculadas desde $298 \mathrm{~K}$ até a sua temperatura crítica. A Figura também traz as curvas calculadas pelas Equações 1 e 2.

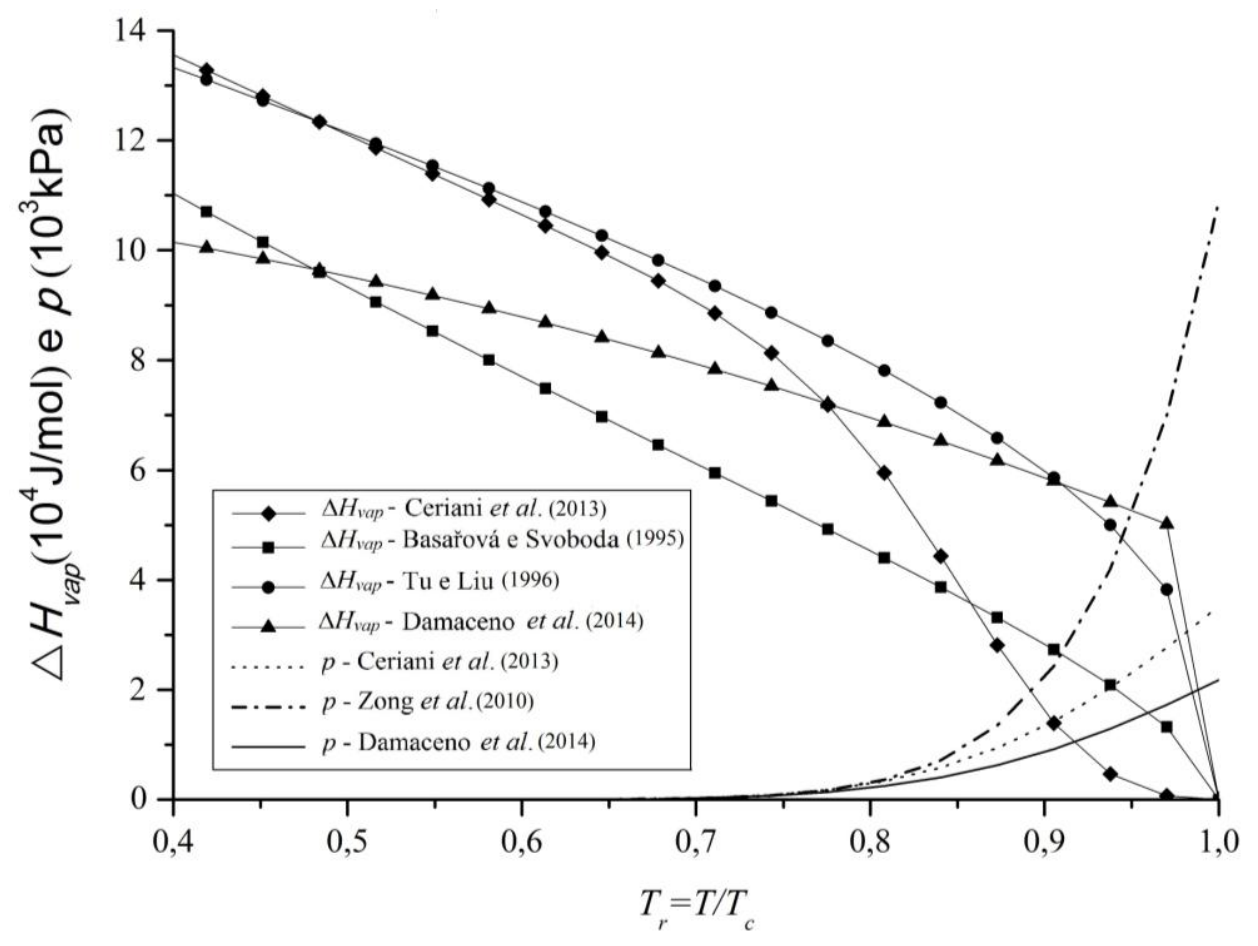

Figura 1 - Curvas de pressão de vapor $(p)$ e de entalpia de vaporização $\left(\Delta H_{v a p}\right)$ para a monocaprilina em função da $T_{r}$, conforme cada modelo 


\section{CONCLUSÃO}

Predizer uma dada propriedade termofísica com alta acurácia é fundamental no desenvolvimento de processos químicos. Nesse trabalho foram selecionadas três propriedades termofísicas importantes de acilgliceróis parciais: pressão de vapor (temperatura de ebulição), entalpia de vaporização e temperatura normal de ebulição, e testados diferentes modelos preditivos baseados no conceito de contribuição de grupos e/ou fragmentos. Para a $T_{n b}$, o modelo de Joback e Reid (1987) apresentou desvio relativo de 26,39 \%. O modelo de Zong et al. (2010) teve desempenho melhor para a $\Delta H_{v a p}$ a 298,15 K do que o modelo de Marrero e Gani (2001). Em relação à temperatura de ebulição, o modelo de Ceriani et al. (2013) apresentou menores desvios relativos em comparação com Zong et al. (2010). Os modelos de Ceriani et al. (2013), Tu e Liu (1996) predisseram a $\Delta H_{v a p}$ de forma equivalente, e o modelo de Basařová e Svoboda (1995) apresentou maiores discrepâncias.

\section{REFERÊNCIAS}

AMERICAN SOCIETY FOR TESTING AND MATERIALS. ASTM E1782. Standard test method for determining vapor pressure by thermal analysis. Annual Book of ASTM Standards, v. 1402, 2008

BASAŘOVÁ, P.; SVOBODA, V. Prediction of the enthalpy of vaporization by the group contribution method. Fluid Phase Equilib., v. 105, n. 1, p. 27-47, 1995.

CERIANI, R.; GANI, R.; LIU, Y.A. Prediction of vapor pressure and heats of vaporization of edible oil/fat compounds by group contribution. Fluid Phase Equilib., v. 337, p. 53-59, 2013.

CERIANI, R.; GONÇALVES, C.; COUTINHO, J. Prediction of viscosities of fatty compounds and biodiesel by group contribution. Energ. Fuel., v. 25, p. 3712-3717, 2011.

CERIANI, R. Simulação computacional de processos de desodorização e desacidificação de óleos vegetais. Campinas, 2005. Tese de doutorado - FEA UNICAMP. 2005.

CUNICO, L. P.; GUIRARDELLO, R.; CERIANI, R. Estimation of Physical Properties of Vegetable Oils and Biodiesel using Group Contribution Methods. Chem. Eng. Trans., v. 32, p. 535-540, 2013.

DAMACENO, D. S.; MATRICARDE FALlEIRO, R. M.; MEIRELLES, A. J. A.; KRÄHENBÜHL, M. A.; MEIRELLES, A. J. A.; CERIANI, R. Boiling points of short-chain partial acylglycerols and tocopherols at low pressures by the Differential Scanning Calorimetry technique. J. Chem. Eng. Data, DOI: 10.1021/je401080p, 2014.

DÍAZ-TOVAR, C.; GANI, R.; SARUP, B. Lipid technology: Property prediction and process design/analysis in the edible oil and biodiesel industries. Fluid Phase Equilib., v. 302, n. 1-2, p. 284-293, 2011.

JOBACK, K. G.; REID R. C. Estimation of pure-component properties from group-contributions. Chem. Eng. Commun., v. 57, n. 1-6, p. 233-243, 1987.

MARRERO, J.; GANI, R. Group-contribution based estimation of pure component properties. Fluid Phase Equilib., v. 183-184, p. 183-208, 2001.

POLING, B.E.; PRAUSNITZ, J.M.; O’CONNELL, J.P. The properties of gases and liquids. 5. ed., McGraw-Hill, 2004.

TU, C.; LIU, C. Group-contribution estimation of the enthalpy of vaporization of organic compounds. Fluid Phase

Equilib., v. 121, n. 1-2, p. 45-65, 1996

ZONG, L.; RAMANATHAN, S.; CHEN, C. Thermophysical Properties of Mono- and Diglycerides with the Chemical Constituent Fragment Approach. Ind. Eng. Chem. Res., v. 49, n. 11, p. 5479-5484, 2010.

Agradecimentos: R. Ceriani agradece à FAPESP, CAPES e CNPq pelo suporte financeiro e bolsas individuais. 